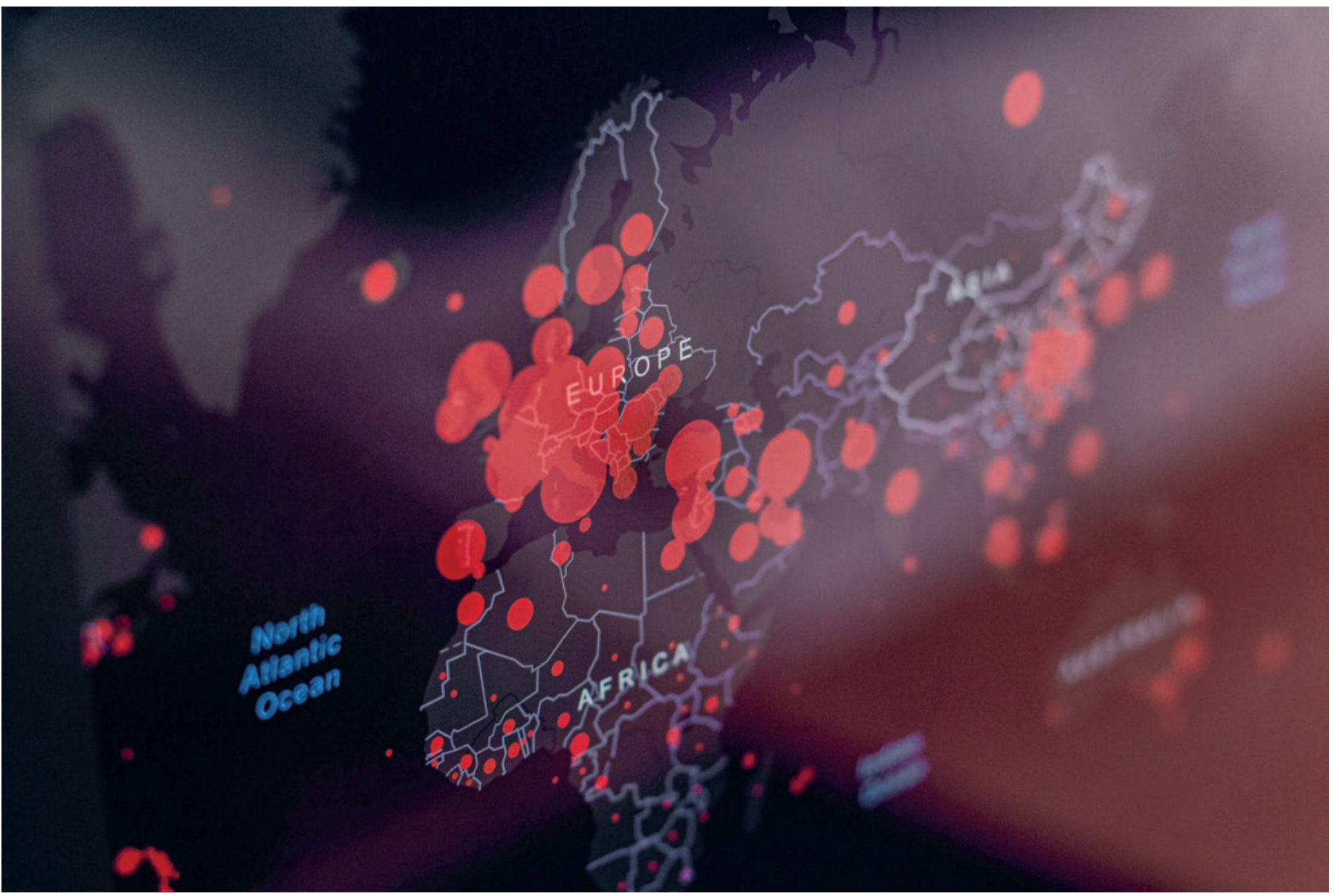

\title{
Une plateforme nationale qui traque le COVID-19 et ses variants
}

Rédaction du Bulletin des médecins suisses

Dans la lutte contre le coronavirus, la Suisse se dote d'une plateforme de surveillance qui traque l'émergence de nouveaux variants. Centralisant les séquences génétiques du COVID-19 récoltées à l'échelle nationale, la «Swiss Pathogen Surveillance Platform» fournit aux autorités une vue globale de la répartition des variants dans le pays. Elle alimente aussi les bases de données internationales servant à la recherche sur le virus.

Alpha, Delta et peut-être bientôt Mu: la Suisse traque l'apparition de nouveaux variants et de leurs chaînes de transmission. Actuellement, le séquençage du génome du virus permet de connaître, à partir d'un test PCR positif, l'identité du variant concerné et son profil génétique complet. Au mois d'août 2021, plus de 5600 séquences ont été analysées par une dizaine de labora- toires académiques ou privés dans toute la Suisse. Ces données ne sont toutefois utiles que si elles sont rapidement mises en rapport les unes avec les autres. Pour répondre à ce besoin de coordination a été créée une infrastructure nationale de surveillance, codirigée par l'Institut Suisse de Bioinformatique (SIB), a annoncé récemment ce dernier dans un communiqué. La Swiss 
Pathogen Surveillance Platform (SPSP) centralise, harmonise et annote les séquences génomiques du SARSCoV-2 provenant des laboratoires en Suisse, ainsi que les données les accompagnant (date du test PCR, mode de prélèvement, raison du séquençage, machine utilisée, lieu du test, genre et âge du patient). Ce nouvel outil vient soutenir la stratégie nationale de surveillance génomique menée par le Centre National de Référence pour les Infections Virales Emergentes (CRIVE) et l'Office fédéral de la santé publique (OFSP), donnant aux autorités une vision d'ensemble et automatisée du séquençage en Suisse.

\section{Suivi de l'épidémie en temps réel}

Trois fois par semaine, la plateforme envoie son rapport de surveillance génomique à l'OFSP qui l'intègre dans ses statistiques, et peut également en recouper les informations avec les données patient dont il dispose sur les hospitalisations, la vaccination, les symptômes au moment du test, etc. Cette stratégie peut servir à identifier qu'une mutation parmi toutes celles observées semble liée à une pathogénicité, ou une résistance au vaccin, plus élevée. Aitana Lebrand, Team Lead Data Science au SIB en charge de la plateforme, explique que «les séquences qui nous arrivent proviennent de la quasi-totalité des cantons suisses: c'est une excellente nouvelle, car cela signifie que de nouveaux variants ont peu de chance de passer sous le radar».

La Suisse est parmi les plus grands contributeurs mondiaux de séquences SARS-CoV-2, après le Royaume-Uni, les Etats-Unis et l'Allemagne.

La plateforme permet aussi de gagner un temps précieux, faisant office de "point d'entrée unique» à une base de données centralisée et standardisée. L'OFSP ne reçoit ainsi plus les rapports de chaque laboratoire dans des formats différents. Ce gain de temps "permet une granularité accrue dans l'analyse des données de séquençage. Ceci nous permet de concentrer nos efforts sur l'adaptation des politiques de santé publique», selon Mirjam Mäusezahl, co-directrice de la section épidémiologie à l'OFSP, citée dans le communiqué.

\section{Coup de fouet à la recherche internationale}

Financée par le Secrétariat d'Etat à la formation, à la recherche et à l'innovation (SEFRI), la nouvelle plateforme transmet aussi les séquences virales, totalement anonymisées, vers des plateformes d'Open Science, telles que le portail COVID-19 européen, pour faire

\section{Swiss Pathogen Surveillance Platform}

Cette plateforme collaborative suit I'approche pluridisciplinaire de One-health et vise, entre autres, à optimiser les résultats en matière de santé humaine. Codirigée par le SIB en collaboration avec les Hôpitaux universitaires de Bâle, Lausanne et Genève, ainsi que les Universités de Berne et de Zurich, elle est hébergée sur l'infrastructure informatique sécurisée du SIB et respecte les normes de sécurité des données du Réseau suisse de santé personnalisée. Le SIB est une organisation à but non lucratif reconnue à l'international et spécialisée en science des données biologiques et biomédicales. II fédère la communauté bioinformatique suisse, soit quelque 800 scientifiques, en encourageant les collaborations et le partage de connaissances.

avancer la recherche internationale. Grâce à ses efforts de partage de données, la Suisse fait désormais partie des plus grands contributeurs de séquences SARSCoV-2 à l'échelle mondiale, après le Royaume-Uni, les Etats-Unis et l'Allemagne. Ces bases de données publiques sont fondamentales pour étudier et comprendre le rôle des variations observées sur la pathogénicité du virus, ses interactions avec les cellules hôtes au moment de l'infection ou encore pour le développement de vaccins et de traitements.

\section{Applicable à d'autres pathogènes}

Pour Christian Lovis, médecin-chef du service de l'information médicale des Hôpitaux universitaires de Genève (HUG), «cette initiative est louable et sera certainement utile pour permettre l'extension à d'autres pathogènes infectieux à l'avenir». La mission initiale de la plateforme du SIB était d'ailleurs de permettre aux spécialistes d'identifier rapidement l'émergence et la propagation de pathogènes et de prendre des mesures précoces pour contenir la transmission en les suivant en temps quasi réel. Avant l'apparition du COVID-19, les bactéries multi-résistantes étaient dans leur radar. "La possibilité d'utiliser SPSP sur le long terme afin de mettre en lien les données génomiques de bactéries ou virus émergents sur le territoire suisse avec des données épidémiologiques est prometteuse pour assurer une réactivité exemplaire de la Suisse en matière de santé publique», affirme dans le communiqué Mirjam Mäusezahl.

\section{Source}

Communiqué de presse du SIB. La Suisse se dote d'une infrastructure nationale pour traquer le COVID-19 et ses variants. 13 octobre 2021.

Crédits photo

KOBU Agency / Unsplash 